\title{
Vehicle Lead-Acid Battery State-of-Charge Meter
}

\author{
Jonathan Scott, Senior Member, IEEE, Kyle Pennington, Member, IEEE, Sergej Schwarz, and Philip Rowe.
}

\begin{abstract}
We describe a state-of-charge, or "residualcapacity" meter for lead-acid batteries that intelligently synthesizes coulometric and terminal-voltage methods in a new algorithm to provide reliable, continuous readout of remaining capacity. Novel electronic circuit design eliminates the need to install a shunt in the vehicle. The meter learns the characteristics of a battery to which it is attached, removing the need for setup, customisation, programming or calibration at time of installation or battery replacement. The meter can thus be installed by unqualified personnel. Initial measurements suggest the design to be robust and accurate.
\end{abstract}

\section{INTRODUCTION}

$\mathbf{E}$ LECTRIC vehicles are expected to have an equivalent of the fuel gauge that appears on the dashboard of vehicles powered by liquid fossil fuels. This proves to be difficult as there is no readily-measured material being consumed as in the case of a fossil-fuel vehicle. A number of approaches have been reported in the literature and a number of products are available on the market, but the complexity of the processes even in the widely-used and well-studied lead-acid battery have thwarted the development of a gauge that finds acceptance and works well in the field.

A number of methods to predict state-of-charge $(\mathrm{SoC})$ or residual capacity have appeared in the literature. Some measure only terminal voltage, some measure cell impedance, some current (charge) conducted, and many use a combination of these. References [1] and [2] both settle on a technique using coulometric measurement weighted for various discharge rates, combined with transient open-circuit voltage to periodically correct for the accumulated error of the Coulomb method. The open-circuit voltage corresponding to full charge is revised to account for ageing effects. The authors of [2] found that this method gave accurate results for electric vehicle applications using lead-acid batteries. The authors of [1] modify the method of [2] only slightly, by removing the transient open-circuit voltage component of the model below 50\% residual capacity where they observed it to be less relaible. The authors of [1] also suggest adaption of the adjustment of capacity with discharge rate, but they measure this for their test batteries and do not elaborate on how the revision of this factor might be achieved.

In [3] the authors present an electric circuit model for an electrochemical battery and show how the open circuit voltage can be estimated based on the battery's terminal voltage and current flow. They suggest that the open circuit voltage can be found when the current is flowing in a parabolic profile. The authors had issues where their extracted open circuit voltage

Jonathan Scott, Kyle Pennington and Philip Rowe are with the Department of Engineering, the University of Waikato, Hamilton, New Zealand. Sergej Schwarz is with the University of Applied Sciences in Regensburg, Germany. e-mail: jonathanscott@ieee.org did not agree forcing them to reset estimators. This method does show potential but is not at a level where it can be practically utilised.

Reference [4] studies the effects of high discharge rates on battery capacity in some detail. The authors propose a weighted coulometric method similar to those of [1] and [2]. Their main contribution is to identify the limitations of various discharge-rate corrections applied in the Coulomb method for predicting residual capacity. They present methods to determine the constants in their equations for given batteries. Their methods have been tested with different discharge patterns for electric vehicles. In conclusion, they do not find a generallyapplicable Coulometric correction technique.

A number of references, after [5] in 2004, report on gauges that integrate current and voltage data and estimate SoC using artificial neural network (ANN) or combinations of similar biologically-inspired methods (dubbed "Soft Computing" in [6]). The implementation reported in [7] represents an application to the Coulomb method explored in [4]. In particular the work addresses the dilemma identified in [4] concerning the application of a model with linear change in Coulometric capacity with discharge current in the case of rapidly-varying discharge current that gave rise to the " $A_{\alpha}$ " and " $A_{r e f}$ " algorithms. This is achieved by using a fuzzy neural network to set the Coulometric correction factor as shown in figure 2 of [7]. This approach is ingenious. The authors of [8] also employ a neural approach with the explicit addition of heuristic rules. They report remarkable success. While these methods are powerful they require significant training, and the training data must include the output variable - the remaining capacitythat is typically only available as an estimate with hindsight. A manufactured gauge would have to be trained, and with data gathered in a controlled way. This challenge is actually noted by the authors of [8]. The ANN approach amounts to an elegant lookup table. The accuracies sought and reported are beyond what would be welcome in practice, but the training amounts to a marketing hurdle. This is naturally unsuitable for a gauge that must be installed in a situation without great preparation or skill.

The approach of refining the Coulomb method through improving the current-dependent correction is pervasive. Radial basis functions and fuzzy logic are applied to this end in [9] and [10] respectively. The authors of [1] and [2] would criticise the approach for its inability to cope with the apparent recovery of capacity that can occur when a battery rests, a kind of memory effect. From the point of view of achieving a gauge with general applicability, it is the training or battery parameter fitting that is the main impediment to this approach.

In contrast [11] describes a method that relies solely on the equilibrium open-circuit EMF for SoC estimation. The authors go to great lengths to estimate this voltage when the battery is 
under load and out of equilibrium through use of terminal voltage, load current and measured battery impedance. Without much ado the authors discount Coulomb methods while using them in characterizing batteries at constant discharge rates, but they do not appear to have considered the approach used in [1] and [2]. As noted in [7], the measurement of impedance requires a serious amount of hardware. Furthermore the authors do not have any measurements using the kind of regime encountered in traction applications such as scooters. It has not been shown that the extra difficulty in using impedance makes the approach in [11] any better than that in [1].

The state-space battery model employed in [12] adds the elegant physical observation that energy from cell reactions delivering current that is not delivered to the user must contribute to heating of the battery. It is hard to use this effectively because a detailed thermal model is needed, and it does not appear to allow for chemical that becomes unavailable from the plates without reacting to contribute charge. [4] Furthermore, temperature sensors must be placed in the electrolyte. In the end the significant computational effort and complicated state-space model produce good results but require excessive hardware and careful parameter fitting.

The authors of [13] propose a model which allows data provided by the manufacturer to be used to predict battery output characteristics for different operating profiles. Their Spice simulation results showed that the model does provide a reasonable representation of the battery terminal voltage for different discharge conditions. However, the work is mostly aimed at predicting performance of batteries in a given application based upon their specifications.

In [14] the authors study how various factors effect battery capacity. Their main contribution is to include temperature as a variable. The authors do not go into detail about the proposed mathematical model. They are concerned more with standby power applications than with traction applications, and thus their results are not closely applicable.

In reference [15] the authors use a method of residual capacity estimation involving electrochemical theory. The paper has a detailed literature review on the conventional methods of determining residual capacity. Their proposed method is largely based around the chemistry within each cell and claims to be quite effective. However, the method relies on battery parameters that are difficult to obtain, and therefore it is inapplicable in the case of a compact model implemented without detailed fitting to a particular battery.

\section{PROposed DESIGN}

Anyone who has used an electric scooter or golf cart will attest to the shortcomings of existing commercial products. On the other hand, reports in the literature suggest that reasonable performance is readily possible, and battery-powered computers report their battery's remaining capacity with decent accuracy. We reconcile this paradox by identifying two aspects of effective gauges that prevent their acceptance in electric vehicle situations:

1) The manufacturer is reluctant to fit a shunt purely to allow current measurement by the meter, especially in the case of low cost vehicles such as scooters. Not only does this add cost and complexity, but the shunt will typically have to be sized for the particular application, and the meter may need to be programmed with the shunt resistance.

2) Manufacturers are not equipped to determine characteristics of the batteries they use, even if they remained constant between batches and suppliers, and are thus reluctant or unable to use a meter that must be programmed with specific constants whose meaning they may not understand in any detail.

In this manuscript we report a meter design that implements proven state-of-charge methods, yet requires neither the installation of a shunt, nor the programming of any batteryspecific parameters. We believe that this will open the way to capacity gauges that find commercial and deployment success. We liken the situation to that surrounding the deployment of the first monolithic operational amplifiers in the 1960s: The opamp only became widely successful when a unitygain compensated version was available, for no less trivial reason than designers who could not or were not willing to go to the length of determining a suitable value of the compensation capacitor. Only when the battery gauge is little more complicated to use than a simple voltmeter will it be accepted. We present such a design here.

\section{A. Current Measurement}

Figure 1 depicts a block diagram of the gauge. There are four electrical connections between the gauge and the vehicle battery system. Two of these are the ground and positive power connections, and two lead to an integrator. The circuitry senses current using an extant section of vehicle wiring as a shunt, typically the battery ground strap. Current is sensed by integrating the small voltage dropped across the cable. This requires the integrator to have very low offsets, but this is not difficult for modern opamps, with a chopperstabilized amplifier costing only a couple of dollars in small quantity. [16]

The proper operation of the integrator and pseudo-shunt is readily checked by means of a current sink that is controlled by the small microcontroller that carries out the state-of-charge calculations. The accuracy of the sink and shunt is not critical, since the gauge is intended to read a fractional capacity, not an absolute value in Ampere-hours, for example. This approach has two collateral advantages: The shunt value tends to be automatically scaled with the vehicle design, since a vehicle that draws larger battery currents is cabled using appropriately thick wire to minimise power loss in the interconnection, while a vehicle that expects to draw smaller currents uses lighter gauge cable to save weight and cost. The two integrator connections do not need to be positioned on the cable a particular way around nor is the distance between them critical, since the current sink will tell the microcontroller the polarity and verify that sufficient cable resistance lies between the integrator connections to allow operation. In our experience the integrator connections should be about $300 \mathrm{~mm}$ apart for satisfactory operation. 


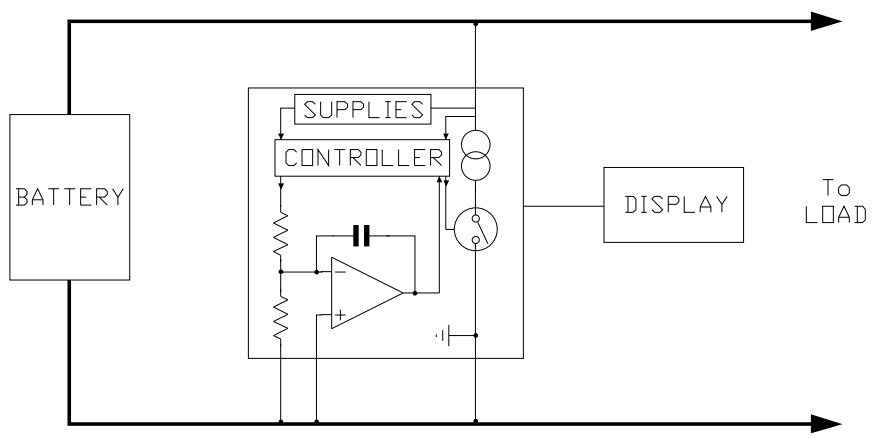

Fig. 1. Block diagram of the gauge associated with a battery and a display. Of interest is the integrator and the switchable current sink controlled by an embedded microcontroller.

\section{B. Operational Algorithm}

The algorithm implemented by the prototype gauge is a periodically-corrected Coulomb method after [2]. The gauge must initially operate in a pure-voltage mode until it deduces the capacity of the battery. Coefficients for both the voltage and the Coulomb methods are refined as opportunity allows during operation.

Specifically, the algorithm operates as follows:

- On cold boot, after charging, or at periodic intervals, the source resistance to the battery is measured using a brief 5-Ampere current pulse. At the same time the current sense polarity is confirmed, and value of the shunt cable is checked to be within acceptable limits.

- The gauge enters a "newborn" mode when first connected to a battery, the battery recovery time constant is set to 600 seconds, and the $a_{1}$ coefficient in the formula that relates open-circuit voltage to state-of-charge is set to 1.5 Volts.

- Whenever the battery's open-circuit voltage exceeds $2.2 \mathrm{~V}$ per cell the gauge resets to full-charge status, setting the consumed charge, $Q_{T}$, to zero.

- If a full-charge reset occurred within the last day and the state-of-charge, $S$, is between $40 \%$ and $75 \%$ and the battery is in equilibrium, the meter leaves "newborn" mode and records the total capacity of the battery, $Q_{B}$, based on the charge drawn out since reaching full charge. Simultaneously, the value of $I_{r e f}$ associated with the " $C_{\text {Iref }}$ " used in the coulometric rate correction algorithm is taken as the average of current drawn over any operating intervals between leaving full charge and leaving "newborn" mode. An "operating interval" is considered to be time when current exceeds 1 Ampere.

- To allow for battery ageing, whenever the battery is fully charged and allowed to rest with negligible current flow until equilibrium is reached, the full-charge, open-circuit voltage is used to update the $a_{1}$ coefficient in the formula that relates open-circuit voltage to state-of-charge, taking $11.8 \mathrm{~V}$ as the open-circuit voltage at zero remaining capacity. [3]

- Battery open-circuit voltage is estimated by one of two methods with the following priority:

1) If the battery is in equilibrium with negligible current being drawn, the terminal voltage is the measured open-circuit voltage;

2) If current is being drawn, the open-circuit voltage is approximated using the full-charge source resistance, the terminal voltage, and Ohm's law.

- Whenever the battery reaches equilibrium, and the gauge is not in "newborn" mode, and the state-of-charge determined from the equilibrium open-circuit voltage exceeds $25 \%$, the value of consumed charge, $Q_{T}$, is corrected to remove the accumulated error, and an incremental adjustment is made to the presumed coulometric capacity of the battery, $Q_{B}$. (Thus the meter allows for incremental changes in battery capacity.)

- The state of charge, $S$, is determined from the opencircuit voltage if the gauge is in its "newborn" mode, or if the equilibrium open-circuit voltage is available and the current value of $S$ is greater than $25 \%$. Otherwise it is obtained from the pessimistic " $A_{\text {ref }}$ " Coulomb method from [4], with $\alpha(i)$ approximated as $0.8+0.2(i) / I_{\text {ref }}$ during discharge, and 0.8 otherwise. (In effect, terminal voltage is used when in equilibrium and not close to flat, consumed charge otherwise.)

- The battery is presumed to be in equilibrium when no current has been drawn for more than four time-constants.

- The battery recovery time constant is adjusted whenever it can be estimated from measurements made at the start of a period of equilibrium.

In addition to the algorithm above, it is possible to implement an automatic identification of the total number of cells. This would permit automatic selection between say, a " $12 \mathrm{~V}$ " mode and " $24 \mathrm{~V}$ " mode, so that the same hardware can service two markets, provided there is sufficient resolution in the ADC hardware.

To date the effects of ambient temperature have not been addressed. A number of constants are expected to exhibit temperature-dependence that may be important in extreme circumstances. However, performance in mild climates does not seem to be greatly impacted and many other authors do not include temperature variation. Accounting for temperature is considered to be an option best left until the other aspects of the approach are proven.

\section{Hardware}

Figure 2 gives an idea of the small size and mechanical simplicity possible with the design. Only four electrical connections are required (excluding any meter illumination). The entire circuit can easily be accomodated on a circuit board whose diameter is less than that of typical meters. Output from the main board consists of an analog voltage in the range $0-5 \mathrm{~V}$ with a series resistance of $4700 \Omega$, allowing the direct connection of a moving-coil meter or an electronic display of some sort.

The authors' implementation employs a Microchip 8-bit microcontroller that sells for around US\$1. [17] There is also a small number of routine components including a CMOS chopper opamp. The circuit board in figure 2 shows a 6-pin "in-circuit serial programming" interface, but this would not 

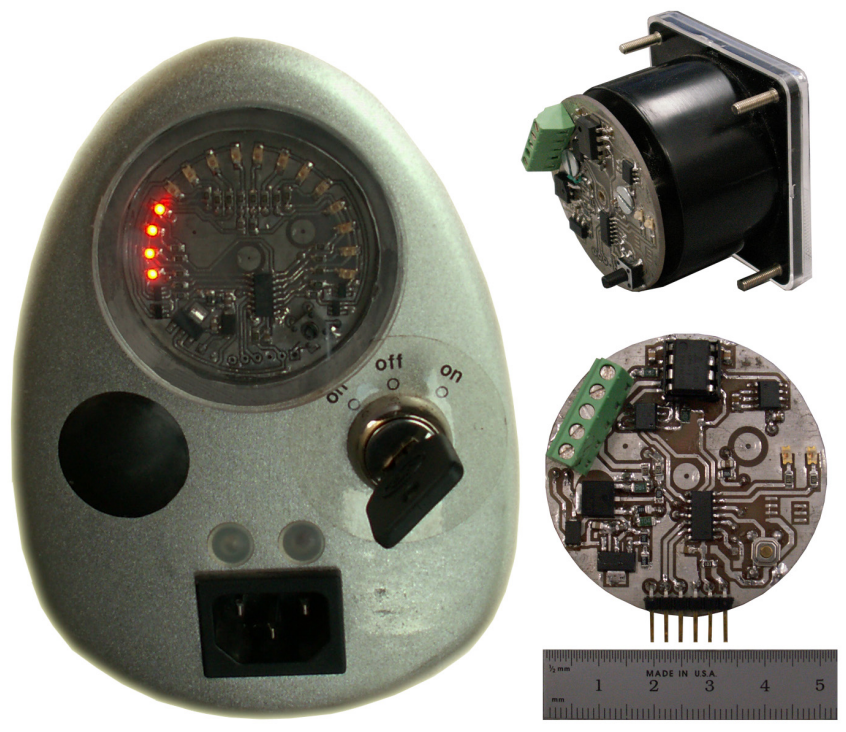

Fig. 2. View of prototype meter mounted in an electric scooter with a solidstate display board, and mounted directly on an analog panel meter. The main printed circuit board is shown along with a scale in centimetres to exemplify its size and simplicity.

be fitted on production units. Installation consists of nothing more than connecting the four wires to power, ground, and the battery earth strap, then pressing the cold-start button. In the event that the two sense wires are connected too closely together or too far apart a signal is given, and the wires need to be repositioned.

\section{Measured Performance}

Figure 3 shows the error in the reading of current on prototype hardware compared with a high-quality bench meter. Constant calibration error arises from error in the inbuilt current calibration pulse. This does not affect operation significantly since the current calibration cancels out in the calculation of SoC. Noise at the low end arises from opamp input offsets, thermocouple voltages, etc. Full scale deflection is set by the controller software and the compensation currents injected into the integrator by the controller, and corresponded to about $1 \mathrm{~V}$ input in this case. The microcontroller-integrator system reads with $10 \%$ accuracy or better across more than 4 orders of magnitude without the use of any special components, solders, or assembly precautions.

Figure 4 shows data gathered using prototype hardware on a new, commercial, lead-acid battery. Figure 5 shows the result of running various algorithms on the example data. It is clear that the use of battery terminal voltage alone is not satisfactory. The methods that use current data yield more convincing results. As suggested in the literature, we have observed that pure Coulomb methods have a tendency to be pessimistic, pure voltage methods a tendency to be erratic. In the example presented here, the periodically-corrected Coulomb algorithm performs best, as expected. It is an amalgam of methods available in the literature and that can be accomodated by

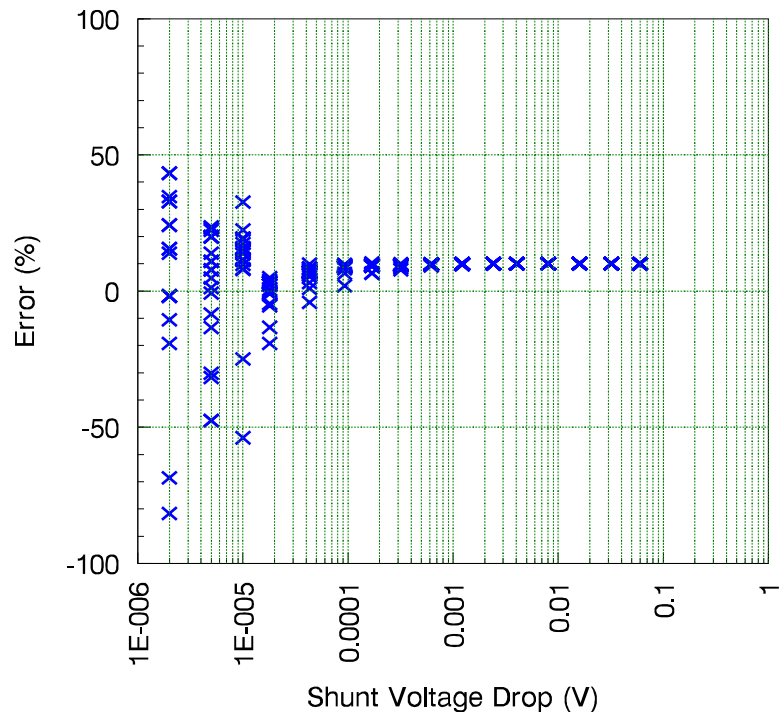

Fig. 3. Error between measured and true current as a function of the voltage across the cable length used as a shunt.

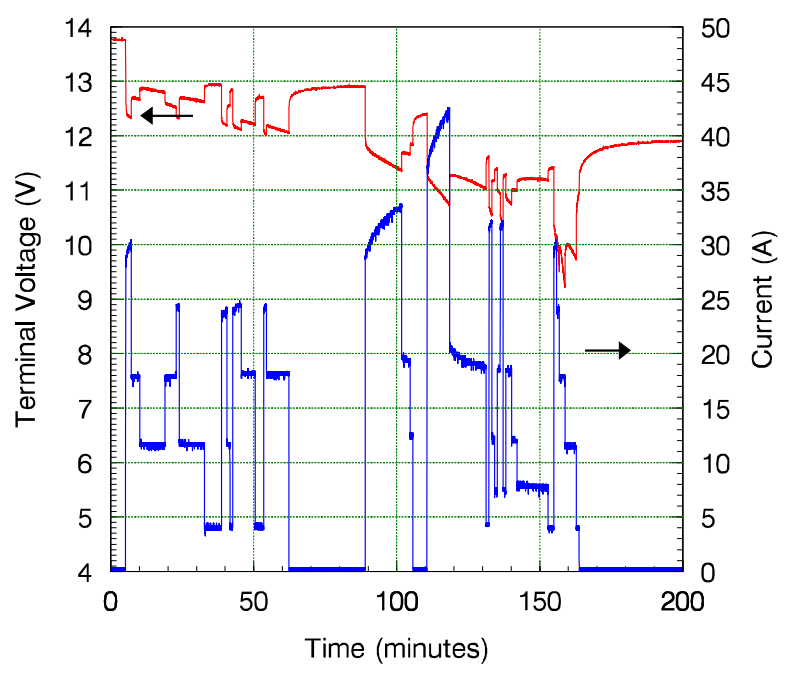

Fig. 4. Typical voltage and current data measured using the prototype meter and used to verify the algorithm code.

our hardware subject to the requirement for autonomous implementation.

It is tempting to ask what might be the "correct answer" for the state-of-charge in any example data set. This is not readily determined, especially without hindsight. [2] Considering the results of figure 5, all methods yield less than zero capacity around 150 minutes, yet power is still being delivered by the battery, so the "correct answer" must lie above zero at that point. Unfortunately, the error is only obvious once the returned state-of-charge falls below zero with power still flowing, and even then it is not obvious at what point in time an algorithm departed from the "correct answer". Not one of the algorithms suggested in the literature is foolproof, and it is possible to devise a load regimen that leads to an optimistic result and another that leads to a pessimistic one. 


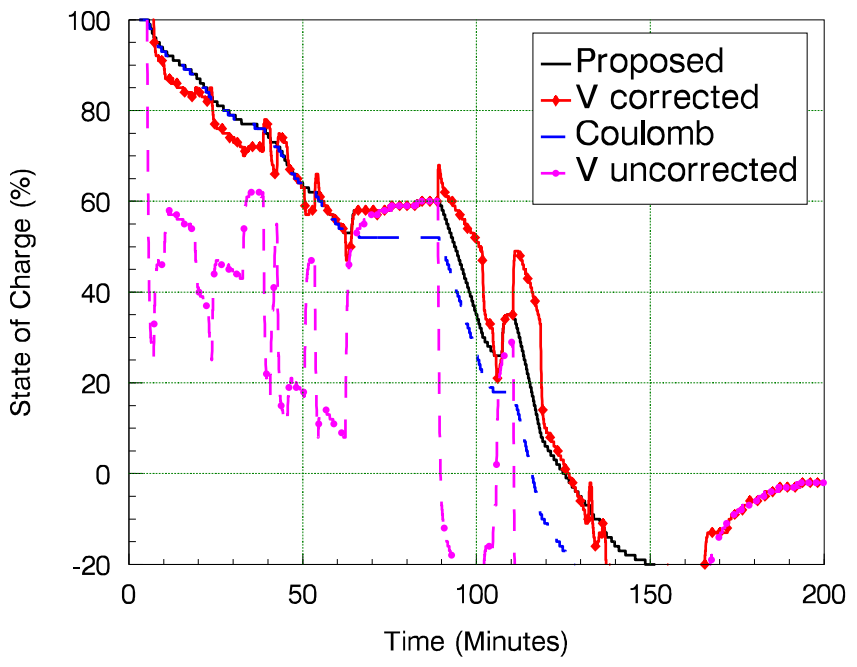

Fig. 5. State-of-charge readout for various methods applied to the data of figure 4 . The plain black line is the proposed method, the plain dashed line is the Coulomb method, the solid line with dots is the voltage method with correction for load current, and the dashed trace with dots is the terminalvoltage method applied by gauges that do not sense current. It is instructive to consider the difference in values that would be returned by the various methods at 15, 100 and 115 minutes of elapsed time. Note the y-axis scale.

\section{ACKNOWLEDGMENT}

The authors wish to acknowledge the technical assistance of Michael Cosgrove and Stewart Finlay.

\section{CONCLUSiOnS}

We have presented a new circuit and algorithm design for a battery state-of-charge gauge. If ease of field use is a determining factor this design could open the way to the widespread use of meters with accuracy comparable to those in fossil-fuel vehicles. We have proven the approach in bench tests. We present a prototype that is being used to replace the gauges in some vehicles. Only more extensive use will establish the practicality of the approach. We encourage the use of the technique presented here in the interests of rigorous testing.

\section{REFERENCES}

[1] Y.-P Yang, J.-J. Liu, and C.-H. Tsai, "Improved estimation of residual capacity of batteries for electric vehicles", Journal of the Chinese Institute of Engineers, Volume 31, Issue 2, 2008 pp313-322.

[2] J. H. Aylor, A. Thieme, and B. W. Johnson, "A Battery State-of-Charge Indicator for Electric Wheelchairs", IEEE Transactions on Industrial Electronics, Volume 39, Issue 5, October 1992 pp398-409.

[3] J. Chiasson and B. Vairamohan, "Estimating the state of charge of a battery", IEEE Transactions on Control Systems Technology, Volume 13, Issue 3, May 2005 pp465-470.

[4] O. Caumont, P. Le Moigne, C. Rombaut, X. Muneret, and P. Lenain, "Energy gauge for lead-acid batteries in electric vehicles", IEEE Transactions on Energy Conversion, Volume 15, Issue 3, September 2000 pp354-360.

[5] Yuang-Shung Lee, Tsung-Yuan Kuo, and Wei-Yen Wang, "Fuzzy Neural Network Genetic Approach to Design the SOC Estimator for Battery Powered Electric Scooter", IEEE Power Electronics Specialists Conference, Aachen, Germany, 2004, pp2759-2765.

[6] Yuang-Shung Lee, Wei-Yen Wang, and Tsung-Yuan Kuo, "Soft Computing for Battery State-of-Charge (BSOC) Estimation in Battery String Systems", IEEE Transactions on Industrial Electronics, Volume 55, no. 1, January 2008, pp229-239.

[7] Der-Tsai Lee, Shaw-Ji Shiah, Chien-Ming Lee, and Ying-Chung Wang, "State-of-Charge Estimation for Electric Scooters by Using Learning Mechanisms", IEEE Transactions on Vehicular Technology, Volume 56, No. 2, March, 2007, pp544-556.

[8] W. X.Shen, C. C. Chan, E. W. C. Lo, and K. T. Chau, "Adaptive NeuryFuzzy Modeling of Battery Residual Capacity for Electric Vehicles", IEEE Transactions on Industrial Electronics, Volume 49, Issue 3, June 2002 pp677-684.

[9] M. Souradip, S. K. Sinha, and K. Muthukumar, "Estimation of State of Charge of Lead Acid Battery using Radial Basis Function", Annual Conference of the IEEE Industrial Electronics Society, 2001.

[10] Souradip Malkhandi, "Fuzzy logic-based learning system and estimation of state-of-charge of lead-acid battery", Engineering Applications of Artificial Intelligence 19, 2006, pp479-485.

[11] Martin Coleman, Chi Kwan Lee, Chunbo Zhu, and William Gerard Hurley, "State-of-Charge Determination From EMF Voltage Estimation: Using Impedance, Terminal Voltage, and Current for Lead-Acid and Lithium-Ion Batteries", IEEE Transactions on Industrial Electronics, Volume 54, Issue 5, October 2007, pp677-684.

[12] Massimo Ceraolo and Giovanni Pede, "Techniques for Estimating the Residual Range of an Electric Vehicle", IEEE Transactions on Vehicular Technology, Volume 50, No. 1, January 2001, pp109-115.

[13] N. K. Medora and A. Kusko, "An Enhanced Dynamic Battery Model of Lead-Acid Batteries Using Manufacturers' Data", Telecommunications Energy Conference, Providence, RI, September 2006 pp1-8.

[14] M. A. Casacca and Z. M. Salameh, "Determination of lead-acid battery capacity via mathematical modeling techniques", IEEE Transactions on Energy Conversion, Volume 7, Issue 3, September 1992 pp442-446.

[15] Rongwen Huang, "Estimation of State-of-Charge of Lead-Acid Batteries Using Electrochemistry Theorem", JSME International Journal Series $C$, Volume 49, Issue 1, 2006 pp265-271.

[16] Intersil Corporation, ICL7650 Datasheet, http://www.intersil.com/ products/deviceinfo.asp?pn=ICL7650S, retrieved June 2009.

[17] Microchip Corporation, http://www.microchip.com/ParamChartSearch/ chart.aspx?branchID $=1002 \&$ mid=10\&lang=en\&pageId $=74, \quad$ retrieved September 2009. 